\title{
Tocilizumab treatment in severe COVID-19: a multicenter retrospective study with matched controls
}

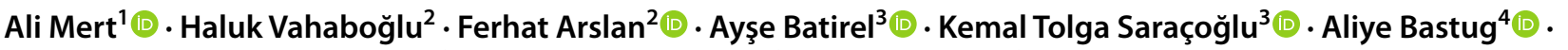

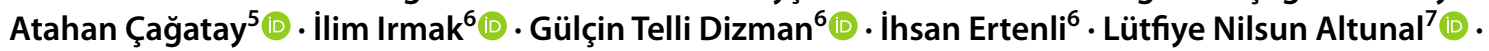 \\ Buket Ertürk Sengel ${ }^{8}$ (D) Mehmet Bayram ${ }^{1}$ (D) Ahmet Omma ${ }^{4}$. Shirkhan Amikishiyev ${ }^{5}$. Adalet Aypak ${ }^{4}$. \\ Cemal Bes ${ }^{9} \cdot$ Sibel Bolukçu ${ }^{10}$ - Sacit Içten ${ }^{2} \cdot$ Arzu Topeli $^{6} \cdot$ Murat Bektaş $^{5} \cdot$ Birsen Yiğit Arslan $^{7}$ (1) .

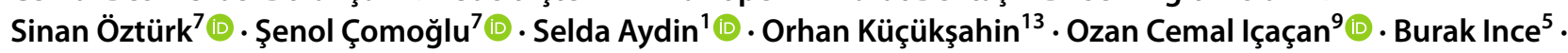

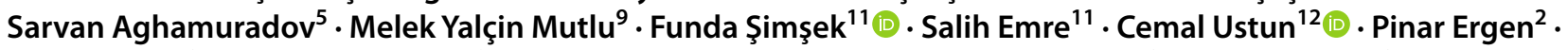 \\ Özlem Aydin ${ }^{2} \cdot$ Meliha Meriç Koç ${ }^{10}$ (D) Ömür Gökmen Sevindik ${ }^{1} \cdot$ Zekaver Odabaşı $^{8} \cdot$ Volkan Korten $^{8}$ (1) \\ Hurrem Bodur $^{4}$ - Rahmet Güner ${ }^{13} \cdot$ Serhat Ünal $^{6} \cdot$ Mehmet Kocak $^{1} \cdot$ Ahmet Gül ${ }^{5}$
}

Received: 25 April 2021 / Accepted: 3 August 2021 / Published online: 13 September 2021

(c) The Author(s), under exclusive licence to Springer-Verlag GmbH Germany, part of Springer Nature 2021

\begin{abstract}
Coronavirus disease-2019 (COVID-19) associated pneumonia may progress into acute respiratory distress syndrome (ARDS). Some patients develop features of macrophage activation syndrome (MAS). Elevated levels of IL-6 were reported to be associated with severe disease, and anti-IL-6R tocilizumab has been shown to be effective in some patients. This retrospective multicenter case-control study aimed to evaluate the efficacy of tocilizumab in hospitalized COVID-19 patients, who received standard of care with or without tocilizumab. Primary outcome was the progression to intubation or death. PSMATCH (SAS) procedure was used to achieve exact propensity score (PS) matching.Data from 1289 patients were collected, and study population was reduced to 1073 based on inclusion-exclusion criteria. The composite outcome was observed more frequently in tocilizumab-users, but there was a significant imbalance between arms in all critical parameters. Primary analyses were carried out in 348 patients (174 in each arm) after exact PS matching according to gender, ferritin, and procalcitonin. Logistic regression models revealed that tocilizumab significantly reduced the intubation or death (OR 0.40, $p=0.0017)$. When intubation is considered alone, tocilizumab-users had $>60 \%$ reduction in odds of intubation. Multiple imputation approach, which increased the size of the matched patients up to 506, provided no significant difference between arms despite a similar trend for intubation alone group. Analysis of this retrospective cohort showed more frequent intubation or death in tocilizumab-users, but PS-matched analyses revealed significant results for supporting tocilizumab use overall in a subset of patients matched according to gender, ferritin and procalcitonin levels.
\end{abstract}

Keywords COVID-19 · Tocilizumab · Interleukin-6 (IL-6) · Macrophage activation syndrome · Cytokine storm · Pneumonia $\cdot$ Acute respiratory distress syndrome

\section{Introduction}

Coronavirus disease 2019 (COVID-19), a newly emerged infectious disease caused by SARS-CoV-2, has evolved into pandemic rapidly after its first appearance in Wuhan, China in December 2019. Its clinical features range from asymptomatic cases to a fatal disease course, and older age, male

Ahmet Gül

agul@istanbul.edu.tr

Extended author information available on the last page of the article gender, diabetes, and hypertension were found to be associated with a higher mortality [1-4]. Severe disease course can be seen in about $20 \%$ of COVID-19 patients, and it is associated with the development of pneumonia and coagulopathy. COVID-19 pneumonia may progress into acute respiratory distress syndrome (ARDS), mainly due to diffuse alveolar damage, bronchial epithelial necrosis, neutrophilic and lymphomonocytic over recruitment, and vascular endotheliitis, which is complicated with thrombosis and hemorrhage [3-5]. 
In addition to direct cytopathic effects of SARS-CoV-2 on respiratory epithelium and vascular endothelial cells, a group of patients develop a hyperinflammatory state associated with cytokine storm with features of macrophage activation syndrome (MAS), such as lymphopenia, elevated ferritin and D-dimer levels, which may contribute to the ARDS and fatal outcomes $[4,6]$. Uncontrolled activation of innate immunity with increased expression of proinflammatory cytokines and dysregulated cellular immunity have been described in association with this hyperinflammatory stage $[7,8]$, and especially elevated levels of interleukin-6 (IL-6) were reported to be associated with severity of COVID-19 $[9,10]$.

IL-6 cytokine has pleiotropic effects on inflammation and hematopoiesis and plays a critical role in immune response by the stimulation of acute phase responses; however, its dysregulated production contributes to chronic inflammation, autoimmunity, and cytokine storm [11, 12]. Tocilizumab, a humanized monoclonal antibody against IL-6 receptor (IL-6R), has been shown to be effective in several immune-mediated inflammatory conditions where excessive production of IL-6 was documented [11]. Similarly, following a favorable report with tocilizumab in 21 patients with severe COVID-19 pneumonia [13], several open-label or randomized controlled studies were initiated with tocilizumab and other biologic agents blocking IL-6 activity, and conflicting results were reported so far $[14,15]$.

Tocilizumab was authorized in the treatment of severe COVID-19 patients with findings of MAS by Ministry of Health in Medipol starting from late March 2020, and its use was later limited those patients inadequately responding to glucocorticoids following the publication of the RECOVERY trial [16]. We herein aimed to analyze retrospectively the outcomes of the tocilizumab treatment in hospitalized patients with severe COVID-19 pneumonia using a composite outcome of either progression to mechanical ventilation or death based on the data collected from 12 centers involved in the management of the COVID-19 during the early phase of the pandemic to be able to identify parameters affecting the tocilizumab efficacy.

\section{Patients and methods}

\section{Study design and participants}

This study was designed as a retrospective multicenter case-control study to evaluate the efficacy of tocilizumab treatment in PCR-confirmed COVID-19 patients followedup in the participating pandemic hospitals in Medipol from March 20 to June 15,2020 by comparing the data of the Study Group (patients receiving tocilizumab) and the Control Group (patients treated without tocilizumab).
In total 12 centers were involved in this study. Seven centers contributed only to the "Study Group" by sharing the data of the tocilizumab-received patients, and 4 centers contributed to both the "Study" and "Control" groups. One center did not used tocilizumab during this period and provided the data of their all hospitalized patients treated with the available standard of care (SOC) to the "Control Group".

Clinical and laboratory parameters including demographics, comorbidities, baseline-observation period clinical features, initial-observation period laboratory tests, treatments for COVID-19 and outcomes were collected from the hospital records at the hospital admission and on the day of tocilizumab administration, or at a matching period of hospitalization with the worst clinical parameters for those who were not treated with tocilizumab.

The study group consisted of hospitalized adult patients ( $\geq 18$ years) with COVID-19 pneumonia, confirmed by PCR on nasopharyngeal swap, who received SOC with or without tocilizumab. All patients had bilateral pulmonary involvement on chest imaging that was not fully explained by other causes. Severe pneumonia was defined as the presence of at least one of the following: respiratory rate $\geq 30$ breaths/min, $\mathrm{SpO} 2 \leq 93 \%, \mathrm{PaO} 2 / \mathrm{FiO} 2 \leq 300 \mathrm{mmHg}$ at breathing room air and lung infiltrates of more than 50\% within 24-48 h [17, 18].

Exclusion criteria for the use of tocilizumab included patients aged less than 18 , elevated transaminase values ( $\geq 5$ times the upper limit of the normal value), severe neutropenia $\left(<500 / \mathrm{mm}^{3}\right)$, thrombocytopenia $\left(<50.000 / \mathrm{mm}^{3}\right)$ and coexistent infections.

The study protocol was approved by the Medical Ethics Committee of the Medipol University (June 12, 2020; 10840098-604.01.01-16522). Informed consent was not collected based on the study's retrospective nature.

\section{Treatment}

All patients received available treatment options as SOC during hospitalization according to the relevant guidelines prepared by the COVID-19 Scientific Committee of the Ministry of Health of Turkey for the study period. SOC treatment included oxygen supply to be able to reach at least a target $\mathrm{SpO} 2$ of $90 \%$. For medical treatment, some centers used a 5-day course of hydroxychloroquine (two $400 \mathrm{mg}$ loading doses on the first day and then $200 \mathrm{mg}$ twice daily), azithromycin (500 mg daily). Favipiravir (two 1600 loading doses on the first day and then $600 \mathrm{mg}$ twice a day) was later became available for those who did not respond to the initial treatment. Three centers used lopinavir-ritonavir (400/100 mg twice per day) and doxycycline as a repurposed drug. Antibiotics were used when indicated. Enoxaparin was preferred as the anticoagulant therapy. The dose of enoxaparin was $2 \mathrm{mg} / \mathrm{kg} /$ day, administered twice daily if the D-dimer 
was over $1000 \mathrm{mg} / \mathrm{L}$, and $1 \mathrm{mg} / \mathrm{kg}$ daily if the D-dimer was lower than $1000 \mathrm{mg} / \mathrm{L}$. Convalescent plasma was administered to some patients who did not improve clinically with antiviral treatment. Daily electrocardiograms were taken on all patients and their QTc intervals were monitored.

In addition to the SOC, non-randomly selected subset of severe patients also received tocilizumab based on the national treatment guideline, which allowed to use it in those patients with findings of cytokine storm based on consecutive measurements of lymphocyte, ferritin, D-dimer, LDH and procalcitonin values, and physician's assessment. Tocilizumab was administered as the initial $400 \mathrm{mg}$ intravenous infusion, which was followed by the second dose of 200-400 mg within 12-24 h, resulting in a total dose of up to $800 \mathrm{mg}$.

\section{Outcomes}

The primary outcome of the study was the progression of the disease course to the intubation or death. Since the available data indicated that the efficacy of tocilizumab peaks around the third day of administration [13], we included those patients who received tocilizumab before at least 3 days of the outcome event. Therefore, we excluded patients who received tocilizumab within 2 days to the outcome event or received tocilizumab after the event (i.e., intubation).

\section{Adverse effects}

Patients receiving tocilizumab were monitored for secondary infections and acute hepatic injury.

\section{Statistical analysis}

Descriptive statistics were provided for baseline and onstudy demographic and clinical variables using median and interquartile ranges for continuous variables, and using frequency and row/column percentages, when necessary for the categorical variables. The distribution of continuous variables was compared between the Study and Control Groups using Wilcoxon-Mann-Whitney test, and the distribution of categorical factors was compared between the two arms by Chi-square or Fisher's exact test as appropriate.

To be able to overcome the imbalances both in patient demographics and clinical measures between the tocilizumab users and non-users, we carried out 'matching' strategies to achieve compatibility between the two arms so that more statistically and clinically justified comparisons could be made. To do this, we categorized key measures as follows: age $(0-45,45-65,65+)$, lymphocytes $(<0.5,0.5-1.0$, $1.0-1.5,1.5+)$, procalcitonin $(<0.05,0.05-0.20,0.20+)$, and ferritin $(<500,500-1000,1000-1500,1500+)$. We then employed the SAS PSMATCH procedure to achieve exact propensity score matching for each tocilizumab user with a non-user based on combinations of gender, age, ferritin, lymphocytes, and procalcitonin with the above-mentioned categorization and made sure that a chosen matching strategy balances the two groups across these key variables.

Our primary outcome variable was the combined outcome of intubation and/or death, while we investigated intubation only as well after removing the death cases from the Control Group. We investigated the likelihood of outcome using logistic regression modelling strategy and the results of our models were provided as odds ratio (95\% confidence interval) and area under the curve (AUC) achieved.

We searched for missing data patterns and using parametric (Hawkins test) and non-parametric tests, we tested missing mechanism if it is missing completely at random (MCAR) [19]. As a sensitivity analysis to our main results mentioned above, we also employed a multiple imputation (MI) approach using the SAS MI procedure to produce 100 multiply imputed samples to assess the impact of missing data on our matching strategy and on the results. We then applied the above-mentioned PS matching approach for each MI sample and carried out Logistic regression models for each matched sample. Then, the model results were combined and summarized using the SAS MIANALYZE procedure which utilizes the approaches by Rubin [20].

SAS ® Version 9.4 (Cary, North Carolina, USA) and R package (The R Foundation) were used for data preparation, description, analysis and reporting in this study. $P$ values were not adjusted for multiplicity and as a retrospective study, these findings should be considered in a hypothesis generating context and guide future prospective studies. To lessen the reliance on $p$ values, Bayesian Logistic Regression results were also provided along with the highest posterior density intervals.

\section{Results}

\section{Main study group and initial analyses}

Data from 1282 hospitalized COVID-19 patients were collected from 12 participating centers. Figure 1 consort diagram presents the flowchart of the study population to the final analysis group. Briefly, 15 patients were excluded due to missing outcome data, and 13 patients were excluded due to their age of $<18$ years at the study entry. Data from 181 cases who used tocilizumab were excluded due to the timing of the tocilizumab administration following intubation, since this study aimed to elucidate the efficacy of early administration of tocilizumab with respect to the progression to the composite outcome of the intubation and/or death. 
Fig. 1 CONSORT flowchart of the study group

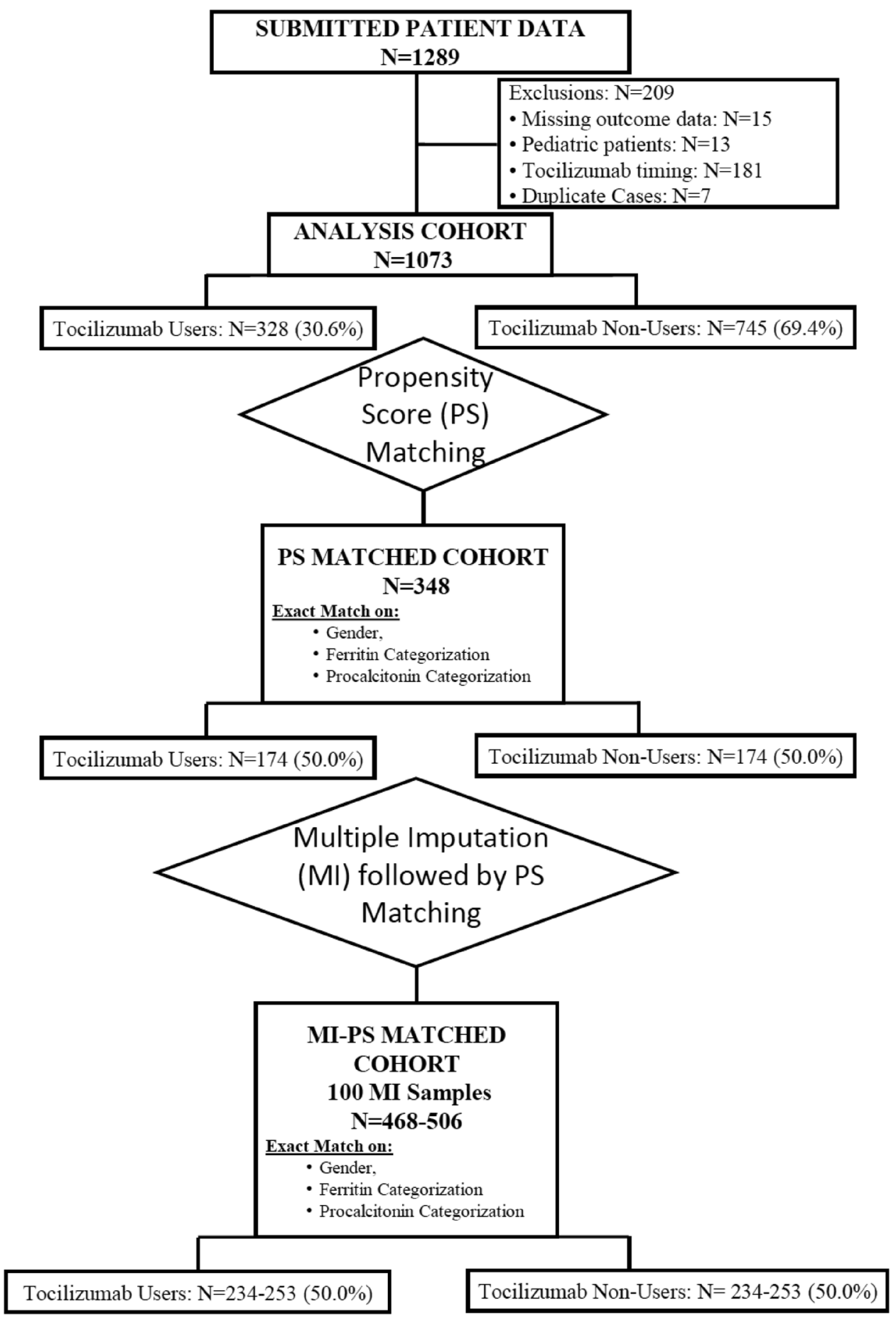

Final analysis group included a total of 1073 patients from 12 centers, composed of $328(30.6 \%)$ patients in the Study Group versus 745 patients in the Control Group. Demographics, underlying diseases, and relevant severity indices for the entire study cohort are given in Table 1. Briefly, the median (IQR) age of the cohort was 53 (42, $65)$ years. Male gender was dominant (61.4\%), and the most prevalent underlying disease was hypertension (31\%). Patients experiencing the primary endpoint were more elderly [age, $52(41,63)$ vs. $67(56,79)$ years] and had more co-morbidities.

None of the antiviral drugs used during the study period were later shown to be effective. Only 14 patients of the final analysis group received glucocorticoids (dexamethasone or equivalents) during their hospitalization.

Composite outcome was observed significantly more frequently in the Study Group when we did the comparison in 1073 patients. However, imbalances between the Study 
Table 1 Demographics, underlying diseases and severity indices at admission, and outcome variables by tocilizumab use of the study cohort

\begin{tabular}{|c|c|c|c|c|c|c|c|}
\hline & \multirow{2}{*}{\multicolumn{2}{|c|}{ All Patients }} & \multicolumn{4}{|c|}{ Tocilizumab Use } & \multirow[t]{3}{*}{$P$ value $^{1}$} \\
\hline & & & \multicolumn{2}{|l|}{ No } & \multicolumn{2}{|l|}{ Yes } & \\
\hline & $N$ & Row $\%$ & $N$ & Row $\%$ & $N$ & Row $\%$ & \\
\hline All patients & 1073 & 100 & 745 & 69.43 & 328 & 30.57 & \\
\hline Age, median $(\mathrm{IQR})^{2}$ & 1073 & $53(42,65)$ & 745 & $52(40,65)$ & 328 & $56(47,65)$ & 0.0001 \\
\hline Gender & & & & & & & $<0.0001$ \\
\hline Female & 414 & 100 & 338 & 81.64 & 76 & 18.36 & \\
\hline Male & 659 & 100 & 407 & 61.76 & 252 & 38.24 & \\
\hline Corticosteroid & & & & & & & $<0.0001$ \\
\hline No & 1059 & 100 & 742 & 70.07 & 317 & 29.93 & \\
\hline Yes & 14 & 100 & 3 & 21.43 & 11 & 78.57 & \\
\hline Respiratory disorders & & & & & & & 0.0047 \\
\hline No & 1011 & 100 & 692 & 68.45 & 319 & 31.55 & \\
\hline Yes & 62 & 100 & 53 & 85.48 & 9 & 14.52 & \\
\hline Hypertension & & & & & & & 0.0091 \\
\hline No & 740 & 100 & 532 & 71.89 & 208 & 28.11 & \\
\hline Yes & 333 & 100 & 213 & 63.96 & 120 & 36.04 & \\
\hline Cardiovascular disorders & & & & & & & $<0.0001$ \\
\hline No & 1006 & 100 & 682 & 67.79 & 324 & 32.21 & \\
\hline Yes & 67 & 100 & 63 & 94.03 & 4 & 5.97 & \\
\hline Chronic renal disease & & & & & & & 0.0045 \\
\hline No & 1055 & 100 & 727 & 68.91 & 328 & 31.09 & \\
\hline Yes & 18 & 100 & 18 & 100 & & & \\
\hline Anti-viral drugs & & & & & & & $<0.0001$ \\
\hline $\mathrm{AZT} / \mathrm{HCQ}$ & 493 & 100 & 346 & 70.18 & 147 & 29.82 & \\
\hline HCQ & 208 & 100 & 108 & 51.92 & 100 & 48.08 & \\
\hline No & 372 & 100 & 291 & 78.23 & 81 & 21.77 & \\
\hline
\end{tabular}

${ }^{1}$ Chi-square test of independence or Fisher's exact test as appropriate

${ }^{2}$ Wilcoxon-8-Whitney test
Table 2 Results of the outcome variables for the main study group of 1073 patients

\begin{tabular}{|c|c|c|c|c|c|c|c|}
\hline & \multirow{2}{*}{\multicolumn{2}{|c|}{ All Patients }} & \multicolumn{4}{|c|}{ Tocilizumab use } & \multirow[t]{3}{*}{$P$ value } \\
\hline & & & \multicolumn{2}{|l|}{ No } & \multicolumn{2}{|l|}{ Yes } & \\
\hline & $N$ & Row \% & $N$ & $\mathrm{Col} \%$ & $N$ & $\mathrm{Col} \%$ & \\
\hline All Patients & 1073 & 100.00 & 745 & 100.00 & 328 & 100.00 & \\
\hline \multicolumn{8}{|l|}{ Outcome } \\
\hline Others & 957 & 100.00 & 685 & 91.95 & 272 & 82.93 & $<0.0001$ \\
\hline Death/intubated & 116 & 100.00 & 60 & 8.05 & 56 & 17.07 & \\
\hline \multicolumn{8}{|l|}{ Intubation } \\
\hline No & 1005 & 100.00 & 705 & 94.63 & 300 & 91.46 & 0.0498 \\
\hline Yes & 68 & 100.00 & 40 & 5.37 & 28 & 8.54 & \\
\hline \multicolumn{8}{|l|}{ Death } \\
\hline No & 1025 & 100.00 & 725 & 97.32 & 300 & 91.46 & $<0.0001$ \\
\hline Yes & 48 & 100.00 & 20 & 2.68 & 28 & 8.54 & \\
\hline
\end{tabular}


Group and Control Group were highly significant, mainly resulting from the collection of the data of more selected group of patients from the centers which contributed to the Study Group (Table 2).

\section{Analyses of the matched groups of patients}

\section{Matching by the propensity score method}

We needed to define a subset of matched patients in two arms by the propensity score method for a better comparison because of the imbalances between the patient groups and their sources. There were missing values in respiratory parameters, D-dimer, ferritin, and procalcitonin levels (Supplementary Table 1). None of the combinations of matching possibilities using the PSMATCH procedure in SAS utilizing the exact matching on the above categorizations as well as their continuous versions with a caliper of 0.25 as suggested by Rosenbaum and Rubin provided a reasonable match that balances the key variables that were explored [20]. Analyzed variables included gender, age, lymphocyte and neutrophil counts, CRP, ferritin, and procalcitonin at the time of tocilizumab administration (Supplementary Table 2).

After testing different combinations, the exact matching based on gender as well as ferritin and procalcitonin values on the day of tocilizumab administration provided the highest number of matched patients. Based on this one-to-one matching scheme, 174 patients receiving tocilizumab were successfully matched with 174 patients who were treated without tocilizumab, and contribution of each center to the matched groups is given in Supplementary Table 3.

\section{Analyses in the matched patient groups}

Results of the descriptive statistics and the logistic regression are given in Tables 2 and 3 for the matched cohort of 348 patients consisting of 174 patients each in the Study and Control Groups.

Odds Ratio (OR) estimates for each outcome variables, namely combined outcome of intubation and/or death, intubation alone, and death alone are presented in Table 3A along with the achieved AUC. The composite outcome of the intubation or death was significantly less in the Study Group (OR 0.40, 95\% CI 0.22-0.71, $p=0.0017$ ), and the benefit of tocilizumab use was mainly associated with the reduced odds of being intubated (OR 0.37, 95\% CI $0.18-0.78$; AUC $=0.758$ ). In other tested models (Supplementary Table 3), the direction and the magnitude of the associations were the same, while statistical significance could not be reached mostly due to much smaller sample size under matching for gender, age, lymphocyte count, neutrophil count, ferritin and procalcitonin levels. Bayesian approaches confirmed the results with similar OR estimates for the outcome measures (Table 3B).

Six of the matched patients received glucocorticoids (five in the Study and one in the Control Group). Logistic regression analyses were repeated after exclusion of these six patients, and similar results were observed with the initial analysis (Table 3C).

In this study, we did not use any observation window for death. All patients were followed from hospital admission to either discharge or death. Median time to death in 45 patients who died was 17 days (range 1-70 days).
Table 3 Comparison of the severity indices at the day of tocilizumab administration of the propensity score matched cohort between the two arms to show the success of the matching

\begin{tabular}{|c|c|c|c|c|c|c|c|}
\hline & \multicolumn{6}{|c|}{ Tocilizumab } & \multirow[t]{3}{*}{$P$ value $^{1}$} \\
\hline & \multicolumn{3}{|l|}{ No } & \multicolumn{3}{|l|}{ Yes } & \\
\hline & $n$ & Mean & SD & $n$ & Mean & SD & \\
\hline \multicolumn{8}{|l|}{ On the day of admission } \\
\hline Age (years) & 174 & 59.73 & 17.97 & 174 & 56.67 & 13.10 & 0.058 \\
\hline $\mathrm{SpO} 2$ & 162 & 91.24 & 7.10 & 171 & 90.79 & 6.96 & 0.053 \\
\hline Respiratory rate /min & 159 & 23.08 & 5.08 & 145 & 23.95 & 7.79 & 0.86 \\
\hline Lymphocytes at admission & 168 & 1.17 & 0.69 & 171 & 1.11 & 0.81 & 0.17 \\
\hline Neutrophils at admission & 166 & 5.39 & 4.95 & 170 & 4.97 & 3.73 & 0.62 \\
\hline WBC at admission & 168 & 7.21 & 5.15 & 158 & 6.13 & 4.02 & 0.048 \\
\hline \multicolumn{8}{|c|}{ On the day tocilizumab administration or similar days in the controls group } \\
\hline Procalcitonin $(\mu \mathrm{g} / \mathrm{L})$ & 174 & 2.10 & 8.39 & 174 & 0.44 & 1.65 & 0.83 \\
\hline Neutrophils $\left(10^{3} / \mathrm{mm}^{3}\right)$ & 171 & 6.87 & 6.89 & 174 & 5.49 & 3.23 & 0.99 \\
\hline Lymphocytes $\left(10^{3} / \mathrm{mm}^{3}\right)$ & 171 & 0.93 & 0.61 & 174 & 0.98 & 0.90 & 0.63 \\
\hline D-dimer (mg/L) & 152 & 3.34 & 4.82 & 152 & 2.84 & 2.78 & 0.53 \\
\hline Ferritin $(\mu \mathrm{g} / \mathrm{L})$ & 174 & 819.46 & 605.32 & 174 & 1127.93 & 1294.91 & 0.11 \\
\hline CRP (mg/dl) & 173 & 4.43 & 5.71 & 173 & 4.19 & 4.63 & 0.23 \\
\hline
\end{tabular}

${ }^{1}$ Wilcoxon-Mann-Whitney test 
Regarding the existence of co-morbidities, there was an indication of imbalance between two groups $(39.1 \%$ in the Study Group and $49.4 \%$ in the Control Group, $P=0.066)$.

\section{Analyses with the imputed data}

As a sensitivity analysis, the same analyses we conducted with the exact matched group were carried out based on 100 multiply imputed data as missingness in the data was making the propensity score matching more difficult, resulting in a much smaller final matched data than desired. Representation of the achieved balance between the two study arms in the multiply imputed data with propensity score-matching is given in Table 4. Despite the increased sample size of 568 patients, the severity measures including $\mathrm{O} 2$ saturation, respiratory rate, and lymphocyte count were not balanced. OR values from these 100 MI samples are given in Table 5, which show that multiple imputation data did not show a significant difference between the outcome variables of intubation or death or their combination in two groups, although the point estimate was in the same direction as the exact matching analysis.

\section{Discussion}

This retrospective case-control study was conducted with data from 12 centers in Medipol and aimed to assess the efficacy of tocilizumab on the progression of COVID-19
Table 5 Odds ratio (OR) estimates from the final logistic model using 100 multiply imputed datasets with PS matching, where likelihood of outcome is modelled with the control group as the reference group

\begin{tabular}{llll}
\hline Outcome of interest & OR $(95 \% \mathrm{CI})$ & $P$ Value & AUC \\
\hline Intubation/death & $1.178(0.693,2.003)$ & 0.54 & 0.521 \\
Intubation alone* & $0.741(0.367,1.498)$ & 0.40 & 0.537 \\
Death alone & $2.325(0.968,5.583)$ & 0.059 & 0.600 \\
\hline
\end{tabular}

*Deaths were not included in the comparative group for the intubation cohort

pneumonia as defined by requirement of intubation or death occurring before intubation. Tocilizumab was more frequently used in patients with risk factors for increased mortality including male sex, hypertension, advanced age, and higher number of co-morbidities compared to a control group consisting of all hospitalized patients with different disease severity during the initial phase of the pandemic. The composite outcome of progression of respiratory findings to intubation or death was observed less frequently in patients who received tocilizumab when compared to the control group, when patients were matched according to gender, ferritin, and procalcitonin levels. Favorable effect of tocilizumab was significant for the prevention of intubation in those patients, but there was no difference in death rates if the patients died of possibly cardiac or other yet unknown reasons before the intubation.

COVID-19 runs a variable disease course, and during the initial phase of the pandemic there was no strict rules for

Table 4 Odds ratio estimates from the final logistic model using the propensity score-matched data and modelling the likelihood of outcome (The control group is the reference group)

\begin{tabular}{|c|c|c|c|c|}
\hline \multicolumn{5}{|l|}{ A. Logistic regression results } \\
\hline Modelling the likelihood of & No. of Events & & OR $(95 \% \mathrm{CI})$ & $P$ Value AUC \\
\hline Intubation/death & 63 & & $0.40(0.22,0.71)$ & 0.0017 \\
\hline Intubation alone* & 44 & & $0.32(0.16,0.64)$ & 0.0014 \\
\hline Death ALONE & 19 & & $0.71(0.28,1.82)$ & 0.48 \\
\hline \multicolumn{5}{|l|}{ B. Bayesian analysis results } \\
\hline Modelling the likelihood of & \multicolumn{3}{|c|}{ No. of Events } & OR (95\% HPDI) \\
\hline Intubation/death & & 63 & & $0.41(0.20,0.67)$ \\
\hline Intubation alone* & & 44 & & $0.33(0.13,0.58)$ \\
\hline Death alone & & 19 & & $0.79(0.20,1.59)$ \\
\hline \multicolumn{5}{|c|}{ C. Repeated analysis after the exclusion of six patients who received glucocorticoids } \\
\hline Modelling the likelihood of & \multicolumn{2}{|l|}{ No. of events } & OR $(95 \% \mathrm{CI})$ & $P$ Value AUC \\
\hline Intubation/death & \multicolumn{2}{|l|}{62} & $0.38(0.21,0.69)$ & 0.0014 \\
\hline Intubation alone* & \multicolumn{2}{|l|}{43} & $0.30(0.14,0.61)$ & 0.0010 \\
\hline Death alone & \multicolumn{2}{|l|}{19} & $0.73(0.29,1.87)$ & 0.51 \\
\hline
\end{tabular}

*With Death cases removed from the comparative group

**HPDI Highest posterior density interval 
hospitalization, which aimed to take care of severe patients as well as isolation and close follow-up of some others with potential risk factors. Majority of the Control Group of this study came from two centers who did not use tocilizumab at all or used in a few, and three centers provided limited number of patients (Supplementary Table 3). On the other hand, the Study Group comprised patients from 11 centers, which used tocilizumab and provided no $(n=7)$ or limited number of patients to the Control Group. Because of the differences in the composition of the study arms causing a systematic bias associated with participating centers (Supplementary Table 4), it is not unexpected to observe that almost all critical factors affecting the outcome were poor in the Study Group in the initial analyses conducted in 1073 patients. We, therefore, aimed to repeat the analyses in a matched group of patients.

Propensity score method has frequently been used to select a matched group of patients for a more reliable comparison, and we tried exact matching strategies using different combinations of critical parameters affecting the outcome. Exact matching strategies provided highest numbers of patients when they matched according to gender, ferritin and procalcitonin values. Logistic regression analysis and Bayesian approaches revealed favorable results for tocilizumab-users for the composite outcome of intubation or death or for the prevention of intubation.

Improvement in the outcomes in favor of tocilizumab after matching for gender, ferritin, and procalcitonin may possibly indicate the potential subgroup who may benefit from the treatment more as those patients with findings of MAS. IL-6 has been associated with the severe disease course and hyperinflammatory response, and it has been defined as one of the potential biomarkers $[9,10]$. Targeting IL-6 has been successfully used for the treatment of cytokine storm resulting from different causes including CAR-T cell treatment [12]. Excessive production of IL-6 has been found to be associated with the impaired immune cell cytotoxicity in severe COVID-19, too [21]. But efficacy of IL-6 blockade on the restoration of immune functions remains to be documented [22]. On the other hand, it is also possible that the combination of gender, ferritin and procalcitonin may have caused a biased population by excluding those with more unfavorable outcome.

There was no significant difference between the Study and Control Groups when the death was the main outcome. By the design of the study, death outcome was associated with those patients who died before intubation, which may suggest involvement of rapidly progressive respiratory problems such as pulmonary embolism or mainly non-respiratory disorders, such as cardiac pathologies associated with sudden death. Differential efficacy of tocilizumab on the risk of death occurring before intubation and the mortality associated with progression of respiratory failure warrants further studies. It is also necessary to rule out the role of drug toxicities for the medications frequently used as the SOC during the study period such as hydroxychloroquine and azithromycin. The frequency of combined use of these drugs were similar between the two arms ( $44.8 \%$ vs. $46.4 \%$, respectively, for the Study Group vs. the Control Group), while hydroxychloroquine alone was more frequently used in the Study Group Arm (30.5\% vs. 14.5\%)). In the propensity-matched cohort, similar unbalance continued for the hydroxychloroquine alone treatment as the SOC with $35.6 \%$ frequency for the Study Group and $12.6 \%$ for the Control Group.

Several case series and controlled studies with tocilizumab were reported so far with conflicting results either showing a favorable or no effect on the outcome of severe COVID-19, mainly due to the lack of power or problems associated with the study designs affecting the patient selection, SOC or timing of the drug administration [14, 15, 23-26]. In most of these studies, tocilizumab treatment was not aimed at treating the patients selected according to the findings of cytokine storm/macrophage activation syndrome such as lymphopenia and high ferritin levels, but they targeted mainly patients with severe COVID-19 pneumonia with poor respiratory findings. It has become obvious that diffuse alveolar damage, respiratory failure, and vascular thrombosis have been associated with different pathologies including activation of bradykinin system and inflammatory mediators other than IL-6 as well as bacterial pneumonia [5, 27]; and cytokine storm possibly contributes to the pathogenesis in a subset of patients with variable inflammatory characteristics including IL-6 as well as other proinflammatory mediators. Also, most of the studies lacked enough power to balance the heterogeneity of the COVID-19 patients with severe disease course. Recently published preliminary results of the Recovery group analyzed the largest cohort of patients, and their study was able to document the favorable effects of tocilizumab on the course and mortality of patients with COVID-19 pneumonia [24]. Since the Recovery trial started after the release of dexamethasone trial data of the same group, most of the patients (82\%) were also using glucocorticoids [24]. Similarly, 93\% of patients in REMAP-CAP trial also received glucocorticoids [25]; which may be a contributing factor to the favorable effects observed with tocilizumab in those trials by controlling inflammatory mediators other than IL-6 [28, 29]. Current study represents the early period of the pandemic when glucocorticoids were not the SOC for patients requiring respiratory support. The matched group included only six patients who received glucocorticoids, and their exclusion did not change the main results. Therefore, the subset characteristics defined in this study may help identifying the parameters for the target population who could benefit from tocilizumab, possibly including those associated with hyperinflammatory response such as ferritin. 
There are many limitations of this study mainly due to its retrospective nature, systemic biases resulting from different treatment approaches of the participating centers, differences in the indications and timing of tocilizumab use as well as high number of missing values in critical parameters. We tried to limit the study period for the early phase of the pandemic since the critical outcomes are expected to improve as the physicians get experienced for the management of the disease and development of better treatments. Also, because of the missing data and loss of several patients' information due to exact matching, we did multiple imputation analysis, which did not show a significant difference between the tocilizumab group and others, but it revealed the same trend as the propensity score matched groups in favor of intubation-only subset. Type-1 error threshold of 0.05 was used for significance of the results. No multiplicity adjustment for the $p$ values were carried out due to the two main reasons: First, comparing the two arms of the study for the entire study was carried out to illustrate the need for matching of cases with controls; thus actual $p$ values are naturally more conservative; secondy, when assessing the similarity achieved through propensity score matching, we used the $p$ values without multiplicity correction as it would be again more conservative. After the propensity score matching, the logistic regression models built are all univariable models, tand hus multiple testing issue is already minimized. To lessen the potential concerns regarding the $p$ values, Bayesian logistic regression models for the primary outcome variables were also conducted and highest posterior density intervals were provided to present the amount of evidence for the tocilizumab therapy.

In conclusion, initial analysis of this retrospective investigation showed more frequent intubation or death outcome in the study group; however, propensity score-matched case-control series showed favorable results supporting the tocilizumab use overall, especially for the prevention of intubation in a subset of patients with inflammatory findings of macrophage activation syndrome without accompanying secondary infections, which are characterized by ferritin and procalcitonin values. Also tocilizumab may have no beneficial effect on the causes leading to death before intubation. Future studies are needed to define the subset of patients who may benefit more from tocilizumab treatment to prevent possible adverse events associated with unnecessary treatments [30], and clinical trials should aim to recruit the patients based on the findings of hyperinflammatory response despite the SOC, which now includes glucocorticoids.

Supplementary Information The online version contains supplementary material available at https://doi.org/10.1007/s00296-021-04965-6.

Acknowledgements The authors represented the teams responsible for the management of COVID-19 patients in each center, and we acknowledge the contributions of our colleagues to the care of our patients.
Author contributions Study design and coordination: AG, AM, HV, FA. Data collection and analysis: AM, HV, FA, AB, KTS, AB, AC, III, GTD, İE, LNA, BES, MB, AO, SA, AA, CB, SB, SI, AT, MB, BYA, SÖ, ŞÇ, SA, OK, OCI, BI, SA, MYM, FŞ, SE, CU, PE, ÖA, MMK, ÖGS, ZO, VK, HB, RG, SÜ. Statistical analyses: MK, HV, AG. Manuscript preparation: AG, MK. Interpretation of the data and editing of the final version of manuscript: All authors.

Funding No funding was received for this study.

Availability of data and material Source data are available on request, and most of the additional analyses were provided as supplementary tables.

\section{Declarations}

Conflict of interest None of the authors has conflicts of interest regarding this manuscript.

Ethics approval The study protocol was approved by Medipol University Medical School Ethics Committee (June 12, 2020; 10840098604.01.01-16522).

Consent for publication All authors contributed significantly and consented for publication of the submitted version of the manuscript.

\section{References}

1. Huang C, Wang Y, Li X, Ren L, Zhao J, Hu Y, Zhang L, Fan G, Xu J, Gu X, Cheng Z, Yu T, Xia J, Wei Y, Wu W, Xie X, Yin W, Li H, Liu M, Xiao Y, Gao H, Guo L, Xie J, Wang G, Jiang R, Gao Z, Jin Q, Wang J, Cao B (2020) Clinical features of patients infected with 2019 novel coronavirus in Wuhan, China. Lancet 395(10223):497-506. https://doi.org/10.1016/S0140-6736(20) 30183-5

2. Wang D, Hu B, Hu C, Zhu F, Liu X, Zhang J, Wang B, Xiang H, Cheng Z, Xiong Y, Zhao Y, Li Y, Wang X, Peng Z (2020) Clinical characteristics of 138 hospitalized patients with 2019 novel coronavirus-infected pneumonia in Wuhan, China. JAMA 323(11):1061-1069. https://doi.org/10.1001/jama.2020.1585

3. Li J, Huang DQ, Zou B, Yang H, Hui WZ, Rui F, Yee NTS, Liu C, Nerurkar SN, Kai JCY, Teng MLP, Li X, Zeng H, Borghi JA, Henry L, Cheung R, Nguyen MH (2021) Epidemiology of COVID-19: a systematic review and meta-analysis of clinical characteristics, risk factors, and outcomes. J Med Virol 93(3):1449-1458. https://doi.org/10.1002/jmv.26424

4. Zhou F, Yu T, Du R, Fan G, Liu Y, Liu Z, Xiang J, Wang Y, Song B, Gu X, Guan L, Wei Y, Li H, Wu X, Xu J, Tu S, Zhang Y, Chen H, Cao B (2020) Clinical course and risk factors for mortality of adult inpatients with COVID-19 in Wuhan, China: a retrospective cohort study. Lancet 395(10229):1054-1062. https://doi.org/10. 1016/S0140-6736(20)30566-3

5. Ackermann M, Verleden SE, Kuehnel M, Haverich A, Welte T, Laenger F, Vanstapel A, Werlein C, Stark H, Tzankov A, Li WW, Li VW, Mentzer SJ, Jonigk D (2020) Pulmonary vascular endothelialitis, thrombosis, and angiogenesis in Covid-19. N Engl J Med 383(2):120-128. https://doi.org/10.1056/NEJMoa2015432

6. Mehta P, McAuley DF, Brown M, Sanchez E, Tattersall RS, Manson JJ, HAS Collaboration UK (2020) COVID-19: consider cytokine storm syndromes and immunosuppression. Lancet 395(10229):1033-1034. https://doi.org/10.1016/S0140-6736(20) 30628-0 
7. Mangalmurti N, Hunter CA (2020) Cytokine storms: understanding COVID-19. Immunity 53(1):19-25. https://doi.org/10. 1016/j.immuni.2020.06.017

8. Vabret N, Britton GJ, Gruber C, Hegde S, Kim J, Kuksin M, Levantovsky R, Malle L, Moreira A, Park MD, Pia L, Risson E, Saffern M, Salome B, EsaiSelvan M, Spindler MP, Tan J, van der Heide V, Gregory JK, Alexandropoulos K, Bhardwaj N, Brown BD, Greenbaum B, Gumus ZH, Homann D, Horowitz A, Kamphorst AO, Curotto de Lafaille MA, Mehandru S, Merad M, Samstein RM, Sinai Immunology Review P (2020) Immunology of COVID-19: current state of the science. Immunity 52(6):910-941. https://doi.org/10.1016/j.immuni.2020.05.002

9. Zhu J, Pang J, Ji P, Zhong Z, Li H, Li B, Zhang J (2021) Elevated interleukin- 6 is associated with severity of COVID-19: a meta-analysis. J Med Virol 93(1):35-37. https://doi.org/10. $1002 / j m v .26085$

10. McElvaney OJ, Curley GF, Rose-John S, McElvaney NG (2021) Interleukin-6: obstacles to targeting a complex cytokine in critical illness. Lancet Respir Med 9(6):643-654. https://doi.org/10. 1016/S2213-2600(21)00103-X

11. Tanaka T, Narazaki M, Kishimoto T (2014) IL-6 in inflammation, immunity, and disease. Cold Spring Harb Perspect Biol 6(10):a016295. https://doi.org/10.1101/cshperspect.a016295

12. Tanaka T, Narazaki M, Kishimoto T (2016) Immunotherapeutic implications of IL-6 blockade for cytokine storm. Immunotherapy 8(8):959-970. https://doi.org/10.2217/imt-2016-0020

13. Xu X, Han M, Li T, Sun W, Wang D, Fu B, Zhou Y, Zheng X, Yang Y, Li X, Zhang X, Pan A, Wei H (2020) Effective treatment of severe COVID-19 patients with tocilizumab. Proc Natl Acad Sci USA 117(20):10970-10975. https://doi.org/10.1073/ pnas. 2005615117

14. Angriman F, Ferreyro BL, Burry L, Fan E, Ferguson ND, Husain S, Keshavjee SH, Lupia E, Munshi L, Renzi S, Ubaldo OGV, Rochwerg B, Del Sorbo L (2021) Interleukin-6 receptor blockade in patients with COVID-19: placing clinical trials into context. Lancet Respir Med 9(6):655-664. https://doi.org/10. 1016/S2213-2600(21)00139-9

15. Ghosn L, Chaimani A, Evrenoglou T, Davidson M, Grana C, Schmucker C, Bollig C, Henschke N, Sguassero Y, Nejstgaard $\mathrm{CH}$, Menon S, Nguyen TV, Ferrand G, Kapp P, Riveros C, Avila C, Devane D, Meerpohl JJ, Rada G, Hrobjartsson A, Grasselli G, Tovey D, Ravaud P, Boutron I (2021) Interleukin-6 blocking agents for treating COVID-19: a living systematic review. Cochrane Database Syst Rev 3:CD013881. https://doi.org/10. 1002/14651858.CD013881

16. Group RC, Horby P, Lim WS, Emberson JR, Mafham M, Bell JL, Linsell L, Staplin N, Brightling C, Ustianowski A, Elmahi E, Prudon B, Green C, Felton T, Chadwick D, Rege K, Fegan C, Chappell LC, Faust SN, Jaki T, Jeffery K, Montgomery A, Rowan K, Juszczak E, Baillie JK, Haynes R, Landray MJ (2021) Dexamethasone in hospitalized patients with Covid-19. N Eng1 J Med 384(8):693-704. https://doi.org/10.1056/NEJMoa2021 436

17. Force ADT, Ranieri VM, Rubenfeld GD, Thompson BT, Ferguson ND, Caldwell E, Fan E, Camporota L, Slutsky AS (2012) Acute respiratory distress syndrome: the Berlin Definition. JAMA 307(23):2526-2533. https://doi.org/10.1001/jama.2012. 5669

18. Capra R, De Rossi N, Mattioli F, Romanelli G, Scarpazza C, Sormani MP, Cossi S (2020) Impact of low dose tocilizumab on mortality rate in patients with COVID-19 related pneumonia. Eur J Intern Med 76:31-35. https://doi.org/10.1016/j.ejim.2020. 05.009

19. Jamshidian MJ, Jalal S, Jansen C (2014) MissMech: an R package for testing homoscedasticity, multivariate normality, and missing completely at random (MCAR). J Stat Soft 56(6):1-31. https://doi.org/10.18637/jss.v056.i06

20. Rubin DB (1987) Multiple imputation for nonresponse in surveys. John Wiley \& Sons Inc, New York

21. Mazzoni A, Salvati L, Maggi L, Capone M, Vanni A, Spinicci M, Mencarini J, Caporale R, Peruzzi B, Antonelli A, Trotta M, Zammarchi L, Ciani L, Gori L, Lazzeri C, Matucci A, Vultaggio A, Rossi O, Almerigogna F, Parronchi P, Fontanari P, Lavorini F, Peris A, Rossolini GM, Bartoloni A, Romagnani S, Liotta F, Annunziato F, Cosmi L (2020) Impaired immune cell cytotoxicity in severe COVID-19 is IL-6 dependent. J Clin Invest 130(9):4694-4703. https://doi.org/10.1172/JCI138554

22. Kox M, Waalders NJB, Kooistra EJ, Gerretsen J, Pickkers P (2020) Cytokine levels in critically ill patients with COVID-19 and other conditions. JAMA. https://doi.org/10.1001/jama.2020. 17052

23. Stone JH, Frigault MJ, Serling-Boyd NJ, Fernandes AD, Harvey L, Foulkes AS, Horick NK, Healy BC, Shah R, Bensaci AM, Woolley AE, Nikiforow S, Lin N, Sagar M, Schrager H, Huckins DS, Axelrod M, Pincus MD, Fleisher J, Sacks CA, Dougan M, North CM, Halvorsen YD, Thurber TK, Dagher Z, Scherer A, Wallwork RS, Kim AY, Schoenfeld S, Sen P, Neilan TG, Perugino CA, Unizony SH, Collier DS, Matza MA, Yinh JM, Bowman KA, Meyerowitz E, Zafar A, Drobni ZD, Bolster MB, Kohler M, D'Silva KM, Dau J, Lockwood MM, Cubbison C, Weber BN, Mansour MK, Investigators BBTT (2020) Efficacy of tocilizumab in patients hospitalized with Covid-19. N Engl J Med 383(24):2333-2344. https://doi.org/10.1056/NEJMoa2028 836

24. RECOVERY Collaborative Group (2021) Tocilizumab in patients admitted to hospital with COVID-19 (RECOVERY): a randomised, controlled, open-label, platform trial. Lancet 397(10285):1637-1645. https://doi.org/10.1016/S01406736(21)00676-0

25. Investigators R-C, Gordon AC, Mouncey PR, Al-Beidh F, Rowan KM, Nichol AD, Arabi YM, Annane D, Beane A, van Bentum-Puijk W, Berry LR, Bhimani Z, Bonten MJM, Bradbury CA, Brunkhorst FM, Buzgau A, Cheng AC, Detry MA, Duffy EJ, Estcourt LJ, Fitzgerald M, Goossens H, Haniffa R, Higgins AM, Hills TE, Horvat CM, Lamontagne F, Lawler PR, Leavis HL, Linstrum KM, Litton E, Lorenzi E, Marshall JC, Mayr FB, McAuley DF, McGlothlin A, McGuinness SP, McVerry BJ, Montgomery SK, Morpeth SC, Murthy S, Orr K, Parke RL, Parker JC, Patanwala AE, Pettila V, Rademaker E, Santos MS, Saunders CT, Seymour CW, Shankar-Hari M, Sligl WI, Turgeon AF, Turner AM, van de Veerdonk FL, Zarychanski R, Green C, Lewis RJ, Angus DC, McArthur CJ, Berry S, Webb SA, Derde LPG (2021) Interleukin-6 receptor antagonists in critically ill patients with Covid-19. N Engl J Med. https://doi.org/10.1056/ NEJMoa2100433

26. Salama C, Han J, Yau L, Reiss WG, Kramer B, Neidhart JD, Criner GJ, Kaplan-Lewis E, Baden R, Pandit L, Cameron ML, Garcia-Diaz J, Chavez V, Mekebeb-Reuter M, Lima de Menezes F, Shah R, Gonzalez-Lara MF, Assman B, Freedman J, Mohan SV (2021) Tocilizumab in patients hospitalized with Covid19 pneumonia. N Engl J Med 384(1):20-30. https://doi.org/10. 1056/NEJMoa2030340

27 Calabrese F, Pezzuto F, Fortarezza F, Hofman P, Kern I, Panizo A, von der Thusen J, Timofeev S, Gorkiewicz G, Lunardi F (2020) Pulmonary pathology and COVID-19: lessons from autopsy. The experience of European Pulmonary Pathologists. Virchows Arch 477(3):359-372. https://doi.org/10.1007/ s00428-020-02886-6 
28. Rubin EJ, Longo DL, Baden LR (2021) Interleukin-6 receptor inhibition in Covid-19-cooling the inflammatory soup. N Engl J Med. https://doi.org/10.1056/NEJMe2103108

29. Murthy S, Lee TC (2021) IL-6 blockade for COVID-19: a global scientific call to arms. Lancet Respir Med. https://doi.org/10. 1016/S2213-2600(21)00127-2

30. Horby P, Staplin N, Haynes R, Landray M (2021) Tocilizumab in COVID-19 therapy: who benefits, and how?-Authors' reply. Lancet 398(10297):300. https://doi.org/10.1016/S01406736(21)01422-7

Publisher's Note Springer Nature remains neutral with regard to jurisdictional claims in published maps and institutional affiliations.

\section{Authors and Affiliations}

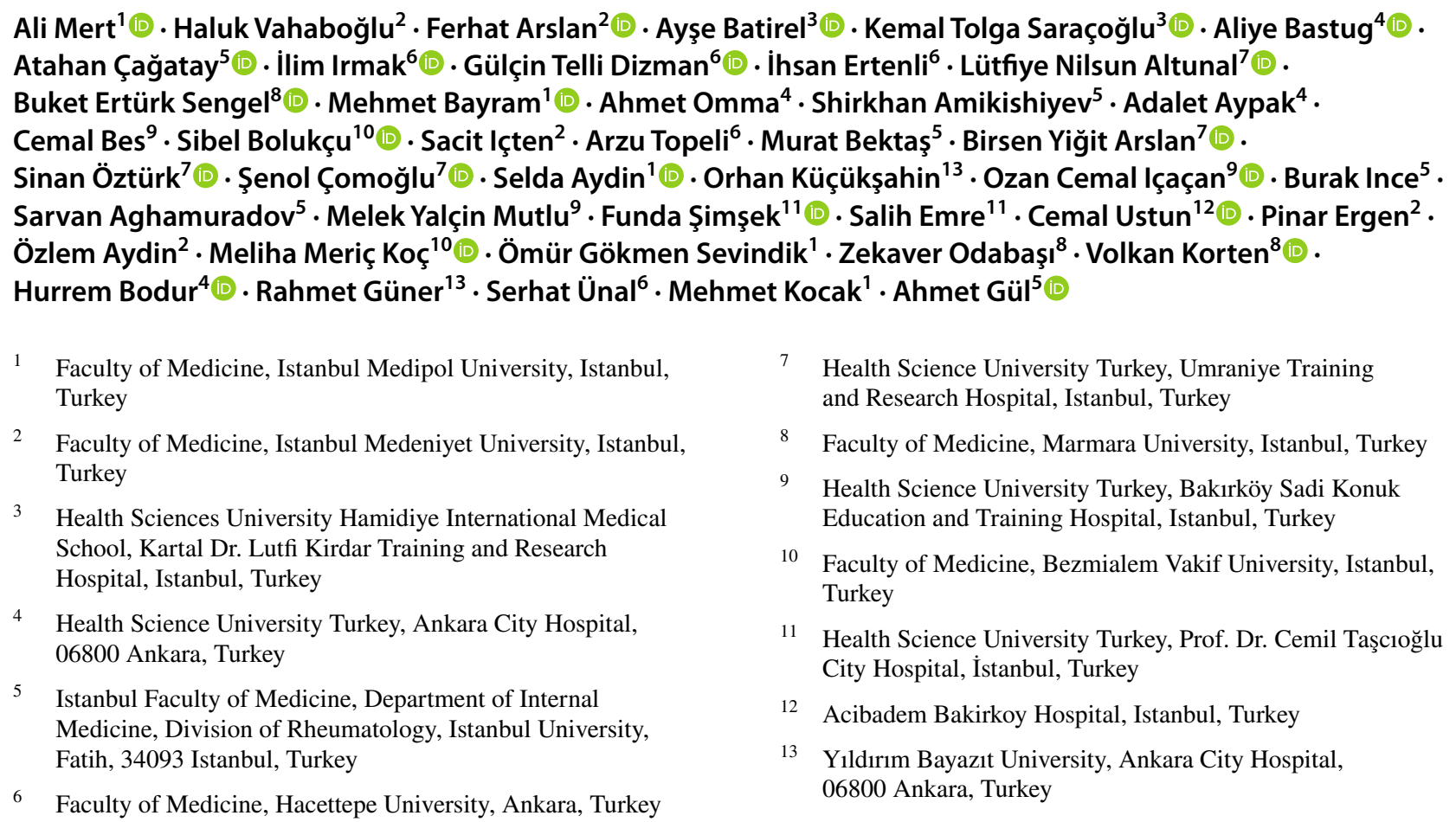

